\title{
Five-beam interference pattern controlled through phases and wave vectors for diamondlike photonic crystals
}

\author{
Yuankun Lin, David Rivera, Zsolt Poole, and Kevin P. Chen
}

\begin{abstract}
We demonstrate, for what is believed to be the first time, the design of diamondlike photonic crystals made by holographic lithography based on five-beam interference. All five beams are launched from the same half-space, and the exposure can easily be realized by a single diffractive optical element. The photonic structure can be constructed through the translation of the interference pattern controlled by the phase shift of laser beams. The proposed holographic lithography is capable of creating series photonic crystals with large photonic bandgaps by adjusting the phase and the wave vector of interfering beams. (C) 2006 Optical Society of America
\end{abstract}

OCIS codes: $\quad 090.0090,260.3160,220.4000$.

\section{Introduction}

Photonic crystals are microstructured materials in which the dielectric constant is periodically modulated on a length scale comparable to the desired electromagnetic wavelength of operation. ${ }^{1,2}$ They hold the promise of numerous applications in integrated optical circuits such as enhancing the performance of semiconductor lasers, waveguides, and all on-chip optical transistors. ${ }^{1-3}$

Holographic lithography has recently been employed to fabricate 3D photonic crystals by exposing a photoresist or polymerizable resin to interference patterns of laser beams. ${ }^{4-16}$ This multibeam interference technique has produced nearly defect-free, nanometerscale structures over large substrate areas. 4,5 Photonic structures are defined in photoresist by isointensity surfaces of interference patterns. ${ }^{9,10}$ In the case of positive resist, the overexposed material is then dissolved away in the postexposure processing. The underexposed region forms a periodic network and acts as a 3D photonic crystal template. The template can then be infiltrated at room temperature with $\mathrm{SiO}_{2}$ and burned away, leaving behind a daughter inverse

Y. Lin (linyk@panam.edu) and D. Rivera are with the Department of Physics and Geology, University of Texas-Pan American, Edinburg, Texas 78541. Z. Poole and K. P. Chen are with the Department of Electrical and Computer Engineering, University of Pittsburgh, Pittsburgh, Pennsylvania 15261.

Received 5 April 2006; revised 11 June 2006; accepted 23 June 2006; posted 28 June 2006 (Doc. ID 69612).

$0003-6935 / 06 / 317971-06 \$ 15.00 / 0$

(C) 2006 Optical Society of America template. ${ }^{17,18}$ Finally, the daughter template is inverted by infiltration with silicon and selective etching of $\mathrm{SiO}_{2} \cdot{ }^{18}$

Holographic lithography allows complete control of the translational symmetry of the photonic crystal and has considerable freedom to design the unit cell. ${ }^{8-15}$ The electrical field of the laser beam can be described by

$$
\mathbf{E}_{i}(\mathbf{r}, t)=\mathbf{E}_{i} \cos \left(k_{i} \cdot r-\omega t+\delta_{i}\right),
$$

where $k$ and $\omega$ are the wave vector and angular frequency of the beam, respectively, $\mathbf{E}$ is the constant of electric field strength, and $\delta$ is the initial phase of the beam. The holographic interference pattern can be designed by controlling interfering beam properties such as electric field strength, polarization, wave vector, and phase. The photonic structure formed through holographic lithography has the translational periodicity determined by the difference between the wave vectors $k_{i}-k_{j}$ of the interfering beams. ${ }^{8-15}$ Therefore, lattice constants of the photonic structure are proportional to the wavelength of the interfering laser beam. The polarization, represented by the electric field vector, determines the motif placed within the unit cell of the photonic lattice. ${ }^{9-13}$ The initial phase difference shifts the interference pattern and changes the motif within the unit cell. ${ }^{11,14,15}$ The laser intensity, exposure time, photoresist preparation, and postexposure development condition will also contribute to the motif of the interference pattern. ${ }^{4,5}$ The photonic structure formed through holographic lithography should have good connectivity 
in both the dielectric and the air component so that the structure is self-supporting and the unwanted photoresist can be dissolved away. ${ }^{11,13}$

So far most published examples of 3D holographic lithography use the four-beam configuration introduced by Campbell et al. ${ }^{4}$ with all four beams coming from the same half-space. However, these fabricated photonic structures have rather small photonic bandgaps. ${ }^{11}$ Recently, people started to design double-exposure holographic lithography to fabricate diamond ${ }^{11,15}$ or diamondlike ${ }^{15}$ photonic structures due to the existence of a large photonic bandgap at lower photonic bands. ${ }^{19}$

Here we study the five-beam interference pattern for the formation of diamondlike tetragonal photonic crystals. All five beams come from the same half-space. It is relatively easier to generate five beams than four beams by using a single diffractive optical element so that the five-beam exposure can easily be realized by a single diffractive optical element. Diamondlike structures are constructed through the translation of the interference pattern controlled by the relative phase difference among interfering beams. The macroscopic structure is controlled through the interference angle among the inferring beams. Detailed photonic bandgap calculations show photonic full bandgaps as large as $22 \%$ of the gap center frequency if the photonic crystal template is converted into silicon.

\section{Theoretical Description}

When the photoresist is exposed to an interference pattern, the interference pattern inside the photoresist side beam also form an angle of $\theta$. The prism is placed on the photoresist surface. An index-matching fluid may be placed between the bottom surface of the topcut prism and the photoresist surface to further reduce the interface effect. All five laser beams come from the same half-space. As shown in Fig. 1(a), beams 1 and 2 propagate in the $x z$ plane and beams 3 and 4 in the $y z$ plane. The fifth one propagates toward the origin along the $z$ axis. These five beams are described by

$$
\begin{aligned}
& \mathbf{E}_{1}(\mathbf{r}, t)=\mathbf{E}_{1} \cos \left[(k \cos \theta) z-(k \sin \theta) x-\omega t+\delta_{1}\right], \\
& \mathbf{E}_{2}(\mathbf{r}, t)=\mathbf{E}_{2} \cos \left[(k \cos \theta) z+(k \sin \theta) x-\omega t+\delta_{2}\right], \\
& \mathbf{E}_{3}(\mathbf{r}, t)=\mathbf{E}_{3} \cos \left[(k \cos \theta) z-(k \sin \theta) y-\omega t+\delta_{3}\right], \\
& \mathbf{E}_{4}(\mathbf{r}, t)=\mathbf{E}_{4} \cos \left[(k \cos \theta) z+(k \sin \theta) y-\omega t+\delta_{4}\right], \\
& \mathbf{E}_{5}(\mathbf{r}, t)=\mathbf{E}_{5} \cos \left(k z-\omega t+\delta_{5}\right) .
\end{aligned}
$$

These five mutually coherent laser beams will generate $3 \mathrm{D}$ interference patterns. The interference pattern is determined by the laser intensity modulation $I$ in $3 \mathrm{D}$ space:

$$
\begin{aligned}
I= & <\sum_{i=1}^{5} \mathrm{E}_{i}^{2}(r, t)>+\sum_{i<j}^{5} \mathrm{E}_{i} \cdot \mathrm{E}_{j} \cos \left[\left(k_{i}-k_{j}\right) \cdot r+\left(\delta_{i}-\delta_{j}\right)\right] \\
= & (1 / 2)\left(\mathrm{E}_{1}^{2}+\mathrm{E}_{2}{ }^{2}+\mathrm{E}_{3}^{2}+\mathrm{E}_{4}{ }^{2}+\mathrm{E}_{5}{ }^{2}\right)+\mathbf{E}_{1} \cdot \mathrm{E}_{2} \cos \left[-(2 k \sin \theta) x+\left(\delta_{1}-\delta_{2}\right)\right] \\
& +\mathbf{E}_{1} \cdot \mathrm{E}_{3} \cos \left[-(k \sin \theta) x+(k \sin \theta) y+\left(\delta_{1}-\delta_{3}\right)\right]+\mathbf{E}_{1} \cdot \mathrm{E}_{4} \cos \left[-(k \sin \theta) x-(k \sin \theta) y+\left(\delta_{1}-\delta_{4}\right)\right] \\
& +\mathbf{E}_{1} \cdot \mathrm{E}_{5} \cos \left[(k \cos \theta-k) z-(k \sin \theta) x+\left(\delta_{1}-\delta_{5}\right)\right]+\mathbf{E}_{2} \cdot \mathrm{E}_{3} \cos \left[(k \sin \theta) x+(k \sin \theta) y+\left(\delta_{2}-\delta_{3}\right)\right] \\
& +\mathbf{E}_{2} \cdot \mathrm{E}_{4} \cos \left[(k \sin \theta) x-(k \sin \theta) y+\left(\delta_{2}-\delta_{4}\right)\right]+\mathbf{E}_{2} \cdot \mathrm{E}_{5} \cos \left[(k \cos \theta-k) z+(k \sin \theta) x+\left(\delta_{2}-\delta_{5}\right)\right] \\
& +\mathbf{E}_{3} \cdot \mathrm{E}_{4} \cos \left[(-2 k \sin \theta) y+\left(\delta_{3}-\delta_{4}\right)\right]+\mathbf{E}_{3} \cdot \mathrm{E}_{5} \cos \left[(k \cos \theta-k) z-(k \sin \theta) y+\left(\delta_{3}-\delta_{5}\right)\right] \\
& +\mathbf{E}_{4} \cdot \mathrm{E}_{5} \cos \left[(k \cos \theta-k) z+(k \sin \theta) y+\left(\delta_{4}-\delta_{5}\right)\right] .
\end{aligned}
$$

will be different from that in air due to the refraction at the air-photoresist interface. A specially designed prism (one top surface and three side surfaces) has been used to realize the desired four-beam interference pattern inside the photoresist. ${ }^{5}$ This prism method is also used for the five-beam interference. Figure 1(a) shows the design of a top-cut prism. The top-cut prism has four side surfaces oriented fourfold symmetrically. The side surface and bottom surface form an angle of $\theta$. Laser beams propagate through the top and side surfaces with their wave vectors perpendicular to the prism surface. Four side beams and one central beam through the top surface are combined and overlap at the bottom surface of the prism. The central beam and
The term $b_{i j}=\mathrm{E}_{i} \cdot \mathrm{E}_{j}$ is determined by both the amplitude and the polarization of the laser beams and can be optimized to improve the contrast of the interference pattern. ${ }^{12}$ Here we focus on the macroscopic and microscopic structures of the interference pattern controlled through the wave vector difference and the relative phase difference, respectively. The polarization of five beams is set assuming that five beams are obtained from a single beam through a diffractive beam splitter and aperture array. ${ }^{16}$ If beams 3,4 , and 5 have linear polarization parallel to the $x$ axis and beams 1 and 2 are linearly polarized in the $x z$ plane with an angle of $\theta$ relative to the $x$ axis, $\mathrm{E}_{1} \cdot \mathrm{E}_{2}$ is equal to $E_{1} E_{2} \cos (2 \theta), \mathrm{E}_{1} \cdot \mathrm{E}_{3}$ is equal to $E_{1} E_{3} \cos (\theta)$, and so 


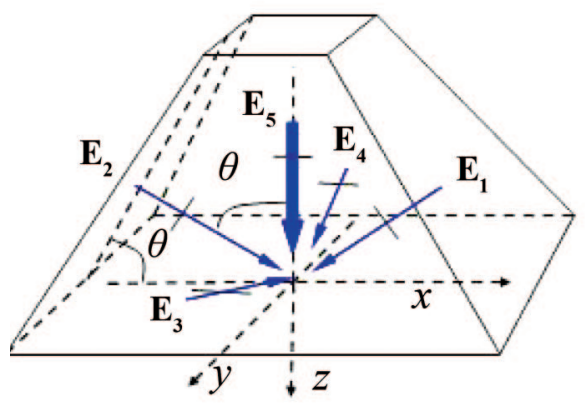

(a)

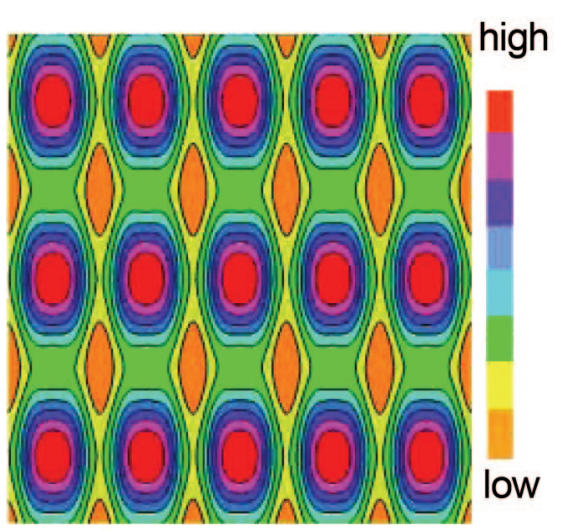

(c)

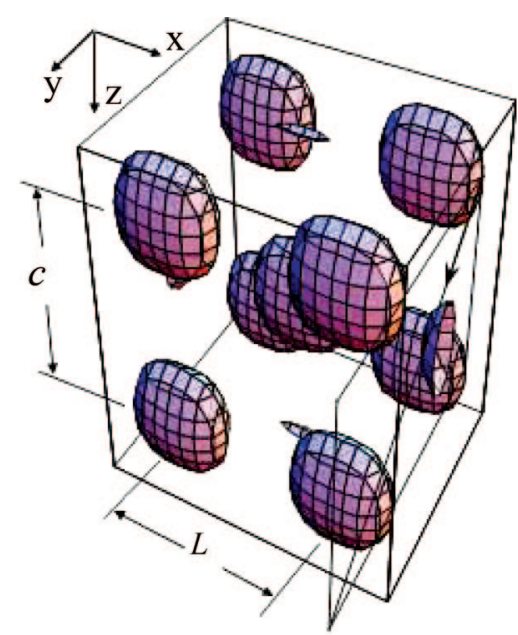

(b)

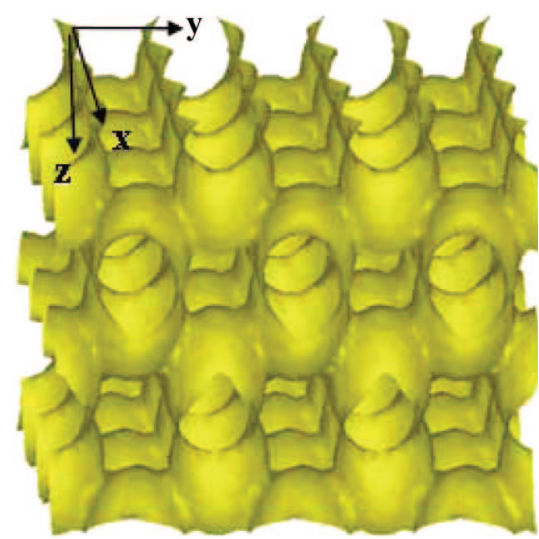

(d)

Fig. 1. (Color online) (a) Beam arrangement through a top-cut prism for the generation of five-beam interference pattern. (b) Five-beam laser interference pattern. The interference pattern is displaced along the arrow to form a diamondlike structure. (c) Cross section of the interference pattern in the $x z$ plane. The color bar indicates the high- and low-intensity parts. (d) Diamondlike structure formed through double exposures.

forth. Assuming that the initial phase difference is a constant (which is true for the coherent laser beam) and the electric field is equal for all five beams, the isointensity surface is shown in Fig. 1(b) for the fivebeam interference pattern in the case of $\theta=58^{\circ}$. Figure 1(c) shows the cross section of the interference pattern in the $x z$ plane. Low-intensity parts of the interference pattern are interconnected, and highintensity parts are isolated from each other. For the proposed diamondlike photonic structure, a low laser dosage will be applied. Then the interference pattern will be translated by controlling the relative phase difference, and the photoresist undergoes a second exposure. For the first exposure, the high intensity part is not connected as shown in Fig. 1(b). After the second exposure, both the low-intensity part and the high-intensity part of the interference pattern are connected. The lattice connectivity can be improved by adjusting the polarization of the interfering beams. The developed negative or positive photoresist becomes a self-supporting photonic crystal template as shown in Fig. 1(d). When SU-8 (negative photoresist, Microchem) is exposed, the overexposed area forms the photonic crystal template. With all the beams coming from the same half-space, this five-beam interference lithography is compatible with conventional photolithography and can be developed on substrates such as silicon, which is opaque to the interfering beam. So researchers can take advantage of the standard tools of the electronics industry for holographic fabrication of $3 \mathrm{D}$ photonic crystals. In the presence of strong reflections from the substrate, the substrate surface can be prepatterned to realize holographic lithography. ${ }^{20}$

In the following we describe how to construct a diamondlike photonic lattice. The interference pattern is periodic in the $z$ direction as well as in the $x$ or $y$ direction. Periodicity $L$ of the interference pattern along the $x$ or $y$ direction is $L=\lambda /(\sin \theta)$. Periodicity $c$ of the interference pattern along the $z$ direction is $c=\lambda /\left[2 \sin ^{2}(\theta / 2)\right]$. For the fcc structure, $c=\sqrt{2} L$. The fcc structure is constructed along the [110], [-110], and [001] directions with a lattice constant of $c$. The 
diagonal direction of the fcc structure is in the direction of [021] as indicated by an arrow in Fig. 1(b). A diamond structure is constructed if the second fcc lattice is translated by $\frac{1}{4}\{0,2 L, c\},=\{0, \sqrt{2} c / 4, c / 4\}$. When $c$ is larger than $\sqrt{2} L$, the structure shown in Fig. 1(b) has a face-centered-tetragonal (fct) symmetry. ${ }^{6}$ When the second fct lattice is translated along [021] by $\{0,0.5 L, 0.25 c\}$, we have a diamondlike structure with fct symmetry as shown in Fig. 1(d). We want to clarify that the indices $\left[n_{1} n_{2} n_{3}\right]$ are based on the $x y z$ coordinate system with scales of $L, L, c$ in the $x, y, z$ directions, respectively.

The translation of the interference pattern along the [021] direction can be induced through the phase shift of interfering beams. Experimentally the phase shift of the laser beam may be produced by phase retarders (such as wave plates and Pockels cells) or by the use of optical delay lines. ${ }^{11,14,15}$ When a phase difference $\left(\rho_{i}-\rho_{j}\right)$ is introduced between interference beams, the interfering term $I_{\text {int }}$ in Eq. (6) becomes

$$
I_{\text {int }}=\sum \mathrm{E}_{i} \cdot \mathrm{E}_{j} \cos \left[\left(k_{i}-k_{j}\right) \cdot r+\left(\rho_{i}-\rho_{j}\right)+\left(\delta_{i}-\delta_{j}\right)\right] .
$$

Such a phase difference between laser beams will translate the interference pattern by $r_{s}$ as described by

$$
I_{\text {int }}=\sum \mathrm{E}_{i} \cdot \mathrm{E}_{j} \cos \left[\left(k_{i}-k_{j}\right) \cdot\left(r+r_{s}\right)+\left(\delta_{i}-\delta_{j}\right)\right] .
$$

The translation $r_{s}$ of the interference pattern is determined by

$$
\left(k_{i}-k_{j}\right) \cdot r_{s}=\rho_{i}-\rho_{j} .
$$

In general, the initial phase difference $\delta_{i}-\delta_{j}$ is a constant if the laser beams are mutually coherent. The $\left(\delta_{i}-\delta_{j}\right)$ will shift the interference pattern relative to the one generated with $\left(\delta_{i}-\delta_{j}\right)=0$. But the initial phase difference will be the same for two exposures. The interference pattern generated by the second exposure needs to be shifted relative to the first one to fabricate the diamondlike photonic crystal. The shifting is produced through the extra phase shift of $\left(\rho_{i}-\rho_{j}\right)$. Specifically, the initial phase difference is zero if all five beams are generated through a single diffractive optical element. ${ }^{16}$ To express the pattern shift as a function of photonic lattice constants, Eq. (7) is rewritten as

$$
\begin{aligned}
I= & (1 / 2)\left(b_{11}+b_{22}+b_{33}+b_{44}+b_{55}\right) \\
& +b_{12} \cos \left[-(4 \pi / L) x+\left(\rho_{1}-\rho_{2}\right)+\left(\delta_{1}-\delta_{2}\right)\right] \\
& +b_{13} \cos \left[-(2 \pi / L) x+(2 \pi / L) y+\left(\rho_{1}-\rho_{3}\right)+\left(\delta_{1}-\delta_{3}\right)\right] \\
& +b_{14} \cos \left[-(2 \pi / L) x-(2 \pi / L) y+\left(\rho_{1}-\rho_{4}\right)+\left(\delta_{1}-\delta_{4}\right)\right] \\
& +b_{15} \cos \left[-(2 \pi / c) z-(2 \pi / L) x+\left(\rho_{1}-\rho_{5}\right)+\left(\delta_{1}-\delta_{5}\right)\right] \\
& +b_{23} \cos \left[(2 \pi / L) x+(2 \pi / L) y+\left(\rho_{2}-\rho_{3}\right)+\left(\delta_{2}-\delta_{3}\right)\right] \\
& +b_{24} \cos \left[(2 \pi / L) x-(2 \pi / L) y+\left(\rho_{2}-\rho_{4}\right)+\left(\delta_{2}-\delta_{4}\right)\right] \\
& +b_{25} \cos \left[-(2 \pi / c) z+(2 \pi / L) x+\left(\rho_{2}-\rho_{5}\right)+\left(\delta_{2}-\delta_{5}\right)\right] \\
& +b_{34} \cos \left[-(4 \pi / L) y+\left(\rho_{3}-\rho_{4}\right)+\left(\delta_{3}-\delta_{4}\right)\right] \\
& +b_{35} \cos \left[-(2 \pi / c) z-(2 \pi / L) y+\left(\rho_{3}-\rho_{5}\right)+\left(\delta_{3}-\delta_{5}\right)\right] \\
& +b_{45} \cos \left[-(2 \pi / c) z+(2 \pi / L) y+\left(\rho_{4}-\rho_{5}\right)+\left(\delta_{4}-\delta_{5}\right)\right] .
\end{aligned}
$$

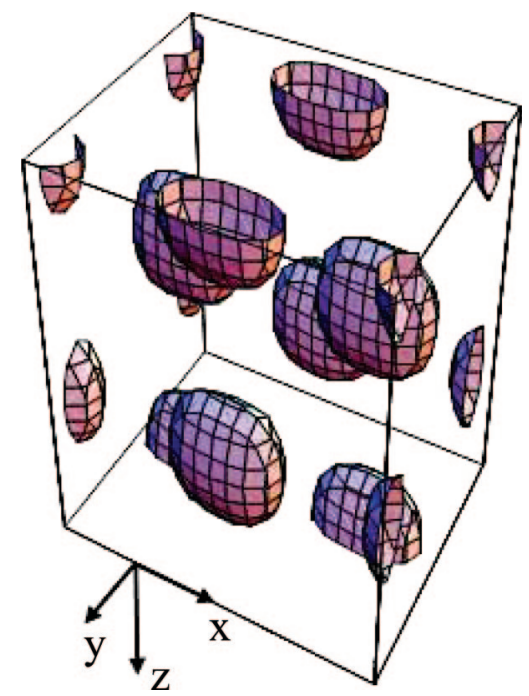

Fig. 2. (Color online) Isointensity surface of the five-beam interference pattern translated through a control of phase shift.

If we want to translate the interference pattern in [021] by $(\phi / 2 \pi)\{0,2 L, c\}$, from Eqs. (10) and (11), phase differences between beams are given by $\rho_{1}-\rho_{2}=0, \rho_{1}-\rho_{3}=2 \phi, \rho_{1}-\rho_{4}=-2 \phi$, and $\rho_{1}-\rho_{5}=-\phi$. Then the phase $\rho_{1}$ of five beams is calculated to be $\rho_{1}=\rho_{2}=2 \phi, \rho_{3}=0, \rho_{4}=4 \phi$, and $\rho_{5}=3 \phi$. If $\phi=0.5 \pi$, the translated interference pattern is expressed as

$$
\begin{aligned}
I= & (1 / 2)\left(b_{11}+b_{22}+b_{33}+b_{44}+b_{55}\right) \\
& +b_{12} \cos \left[-(4 \pi / L) x+\left(\delta_{1}-\delta_{2}\right)\right] \\
& -b_{13} \cos \left[-(2 \pi / L) x+(2 \pi / L) y+\left(\delta_{1}-\delta_{3}\right)\right] \\
& -b_{14} \cos \left[-(2 \pi / L) x-(2 \pi / L) y+\left(\delta_{1}-\delta_{4}\right)\right] \\
& +b_{15} \sin \left[-(2 \pi / c) z-(2 \pi / L) x+\left(\delta_{1}-\delta_{5}\right)\right] \\
& -b_{23} \cos \left[(2 \pi / L) x+(2 \pi / L) y+\left(\delta_{2}-\delta_{3}\right)\right] \\
& -b_{24} \cos \left[(2 \pi / L) x-(2 \pi / L) y+\left(\delta_{2}-\delta_{4}\right)\right] \\
& +b_{25} \sin \left[-(2 \pi / c) z+(2 \pi / L) x+\left(\delta_{2}-\delta_{5}\right)\right] \\
& +b_{34} \cos \left[-(4 \pi / L) y+\left(\delta_{3}-\delta_{4}\right)\right] \\
& -b_{35} \sin \left[-(2 \pi / c) z-(2 \pi / L) y+\left(\delta_{3}-\delta_{5}\right)\right] \\
& -b_{45} \sin \left[-(2 \pi / c) z+(2 \pi / L) y+\left(\delta_{4}-\delta_{5}\right)\right] .
\end{aligned}
$$

The isointensity surface expressed by Eq. (12) is shown in Fig. 2. Compared with Fig. 1(b), the interference pattern is indeed translated by $\{0,0.5 L, 0.25 c\}$. A diamondlike photonic crystal template can be fabricated in the photoresist when the double exposed interference patterns are shifted by $\{0,0.5 L, 0.25 c\}$. To have such translation of $\{0,0.5 L, 0.25 c\}$, beams 1 and 2 must be phase shifted by $\pi$ and beam 5 phase shifted by $1.5 \pi$.

\section{Photonic Bandgap Calculation}

The photonic crystal template must be converted into high refractive index materials to achieve a full bandgap photonic crystal. The details of silicon conversion have been described in Ref. 18. We calculate the photonic bandgap for silicon structures (the high- 
intensity part of the interference pattern is in air and the low intensity part is filled with silicon with a dielectric constant of 11.9) with the MIT PhotonicBands package. ${ }^{21}$ When all the beams come from the same half-space, the interfering angle is expected to be a relatively small value. With the prism method, the interfering angle can be set to be a large value. ${ }^{5}$ To have a fcc structure, the interfering angle should be $70.5^{\circ}$. It is interesting to calculate the photonic bandgap in photonic crystals formed with a smaller interfering angle because it is possible that these crystals could be fabricated through the single diffractive optical element as discussed in Section 4.16 Figure 3(a) shows the photonic band structure for photonic crystals formed through double exposure to the five-beam interference pattern (the dielectric constant of 11.9 is used for silicon, ${ }^{9}$ and the silicon volume fraction is approximately $23 \%$ ). The interference pattern is translated by $\{0,0.5 \mathrm{~L}, 0.25 \mathrm{c}\}$ for the second exposure. Angle $\theta$ between the side surface and the bottom surface of the specially designed prism is set to be $58^{\circ}$ (the $58^{\circ}$ angle is obtained when $c / L=1.8$ ). The first Brillouin surface of the fct lattice is shown in the inset of Fig. 3(a). We want to clarify that the $\lambda_{\text {photon }}$ in the $y$ axis label of Fig. 3(a) is the free-space operating wavelength, not the wavelength of the exposure laser. The band structure shows that a photonic full bandgap exists between the second and the third bands with a bandgap size of $18 \%$ of the gap center frequency.

We calculate the photonic bandgap sizes as functions of translation of the interference pattern along the [021] direction controlled through the phase shift. The calculation is based on photonic structures with $\theta=58^{\circ}$, and the result is shown in Fig. 3(b). The photonic bandgap size has a maximum value when the second interference pattern is translated by a quarter of $\left[(2 L)^{2}+c^{2}\right]^{1 / 2}$ along the arrow as shown in Fig. 1(b). The bandgap size drops when the second interference pattern is shifted other than $\{0,0.5 L, 0.25 c\}$. From Fig. 3(b), we can see that the diamondlike photonic structure is preferred for a larger bandgap size. But the bandgap size is retained above $10 \%$ for small structural deviations from the diamondlike structure during the experiment.

Angle $\theta$ between the side surface and the bottom surface of the prism is related to the wave vector of laser beams that propagate through the side surface [see Fig. 1(a)]. From Eq. (7) we know that both macroscopic (lattice constant) and microscopic (motif) structures of the interference pattern are different for different $\theta$ angles. When angle $\theta$ becomes smaller, the ratio of lattice constants $c / L$ increases although the photonic structure still has fct symmetry. Figure 3(c) shows the optimum photonic bandgap size for the diamondlike structure formed through double exposure at different $\theta$ angles. Photonic crystals can have a bandgap size as large as $22 \%$ of the gap center frequency if it is fabricated through double exposure to the interference pattern formed with a bigger angle $\theta$. The bandgap size drops for the photonic struc-

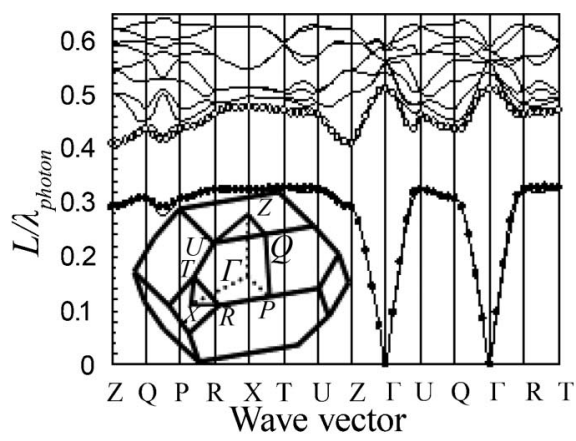

(a)

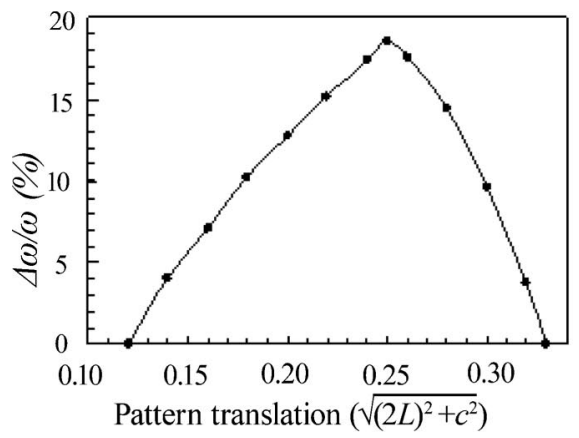

(b)

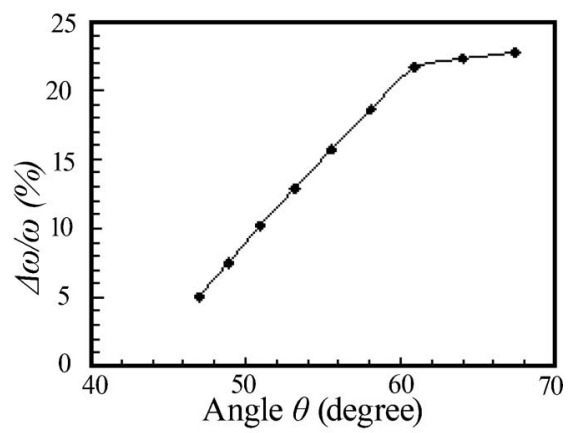

(c)

Fig. 3. (a) Photonic band structure for a diamondlike fct photonic crystal formed through double exposures to the five-beam interference pattern with the interfering angle $\theta=58^{\circ}$. The refractive index of silicon is set to be 3.45 and the silicon volume fraction is $\sim 23 \%$. The position of the high symmetry points together with the irreducible Brillouin zone are shown in the inset. (b) Photonic bandgap sizes versus the translation of the interference pattern controlled through the phase shift. (c) Optimum photonic bandgap sizes as a function of the laser beam interfering angle $\theta$.

ture formed under decreasing angle $\theta$. With different angles $\theta$, one can control the interference pattern and thus control the bandgap size and location.

\section{Discussion}

The holographic lithography technique has been successfully used to fabricate the $3 \mathrm{D}$ photonic crystal. ${ }^{4} \mathrm{~A}$ fabrication strategy that relies on multibeam interference can introduce alignment complexity and inaccuracies due to differences in the optical path length and angles among the interfering beams as well as vibrational instabilities in the optical setup. To improve the 
optical setup by reducing the complexity and instability, diffractive optical elements or phase masks have been introduced to create the interference pattern for the holographic fabrication of photonic crystals.6,7,16 When a single beam goes through a diffractive optics element or a phase mask, the single beam will be diffracted into zeroth-order, first-order, or even higherorder beams. If designed properly, a single optical element can replace a complex optical setup. Another advantage of these single optical element techniques is their compatibility with conventional photolithography so that researchers can take advantage of the standard tools of the electronics industry for the fabrication of $3 \mathrm{D}$ photonic crystals.

It is relatively easier to generate five beams than four beams by using a single diffractive optical element. ${ }^{16}$ Two-dimensional phase masks need to be considered to generate the five beams (one zerothorder plus four first-order diffraction beams). The motif within the unit cell of the photonic crystal is adjustable by controlling the relative intensity of the central laser beam (zeroth-order beam) to the side beams (first-order beams), which is determined by the diffraction efficiency of the phase mask. Diamondlike photonic crystals can be produced through two independent optical exposures with two diffractive optical elements separated by a certain distance. Phase relations between multiple beams can be controlled with almost arbitrary precision by changing only the distance between the applied diffractive optical elements. ${ }^{22}$

\section{Summary}

To the best of our knowledge, we are the first to show that it is possible to design a holographic five-beam interference pattern for the fabrication of diamondlike photonic crystals. All five beams are from the same half-space. We have demonstrated that the proposed holographic lithography is capable of creating photonic structures with large photonic bandgaps by controlling the phase shifts and the wave vectors of interfering beams.

\section{References}

1. S. John, "Strong localization of photons in certain disordered dielectric superlattices," Phys. Rev. Lett. 58, 2486-2489 (1987).

2. E. Yablonovitch, "Inhibited spontaneous emission in solidstate physics and electronics," Phys. Rev. Lett. 58, 2059-2062 (1987).

3. J. D. Joannopoulos, P. R. Villeneuve, and S. H. Fan, "Photonics crystals: putting a new twist on light," Nature 386, 143-147 (1997).

4. M. Campbell, D. N. Sharp, M. T. Harrison, R. G. Denning, and A. J. Turberfield, "Fabrication of photonic crystals for the visible spectrum by holographic lithography," Nature 404, 53-56 (2000).

5. Yu. V. Miklyaev, D. C. Meisel, A. Blanco, G. von Freymann, K. Busch, W. Koch, C. Enkrich, M. Deubel, and M. Wegener, "Three-dimensional face-centered-cubic photonic crystal templates by laser holography: fabrication, optical characteriza- tion, and band-structure calculations," Appl. Phys. Lett. 82, 1284-1286 (2003).

6. Y. Lin, P. R. Herman, and K. Darmawikarta, "Design and holographic fabrication of tetragonal and cubic photonic crystals with phase mask: toward the mass-production of threedimensional photonic crystals," Appl. Phys. Lett. 86, 071117 (2005).

7. Y. Lin, P. R. Herman, and E. L. Abolghasemi, "Proposed singleexposure holographic fabrication of microsphere-type photonic crystal through phase mask techniques," J. Appl. Phys. 97, $096102(2005)$.

8. V. Berger, O. Gauthier-Lafaye, and E. Costard, "Photonic bandgaps and holography," J. Appl. Phys. 82, 60-64 (1997).

9. O. Toader, T. Y. M. Chan, and S. John, "Photonic bandgap architectures for holographic lithography," Phys. Rev. Lett. 92, 043905 (2004).

10. T. Y. M. Chan, O. Toader, and S. John, "Photonic bandgap templating using optical interference lithography," Phys. Rev. E 71, 046605 (2005).

11. D. N. Sharp, A. J. Turberfield, and R. G. Denning, "Holographic photonic crystals with diamond symmetry," Phys. Rev. B 68, 205102 (2003).

12. X. Ao and S. He, "Two-stage design method for realization of photonic bandgap structures with desired symmetries by interference lithography," Opt. Express 12, 978-983 (2004).

13. C. K. Ullal, M. Maldovan, M. Wohlgemuth, C. A. White, S. Yang, and E. L. Thomas, "Triply periodic bicontinuous structures through interference lithography: a level-set approach," J. Opt. Soc. Am. A 20, 948-954 (2003).

14. J. H. Moon, S.-M. Yang, D. J. Pine, and W. Chang, "Multipleexposure holographic lithography with phase shift," Appl. Phys. Lett. 85, 4184-4186 (2004).

15. J. H. Moon, S. Yang, D. J. Pine, and S.-M. Yang, "Translation of interference pattern by phase shift for diamond photonic crystals," Opt. Express 13, 9841-9846 (2005).

16. T. Kondo, S. Matsuo, S. Juodkazis, and H. Misawa, "Femtosecond laser interference technique with diffractive beam splitter for fabrication of three-dimensional photonic crystals," Appl. Phys. Lett. 79, 725-727 (2001).

17. H. Miguez, N. Tetreault, B. Hatton, S. M. Yang, D. Perovic, and G. A. Ozin, "Mechanical stability enhancement by pore size and connectivity control in colloidal crystals by layer-bylayer growth of oxide," Chem. Commun. (Cambridge) 22, 2736-2737 (2002).

18. N. Tereault, G. von Freymann, M. Deubel, M. Hermatschweiler, F. Perez-Willard, S. John, M. Wegener, and G. A. Ozin, "New route to three-dimensional photonic bandgap materials: silicon double inversion of polymer templates," Adv. Mater. 18, 457460 (2006).

19. K. M. Ho, C. T. Chan, and C. M. Soukoulis, "Existence of a photonic gap in periodic dielectric structures," Phys. Rev. Lett. 65, 3152-3155 (1990).

20. M. Trupke, F. Ramirez-Martinez, E. A. Curtis, J. P. Ashmore, S. Eriksson, E. A. Hinds, Z. Moktadir, C. Gollasch, M. Kraft, G. Vijaya Prakash, and J. J. Baumberg, "Pyramidal micromirrors for microsystems and atom chips," Appl. Phys. Lett. 88, 071116 (2006).

21. S. G. Johnson and J. D. Joannopoulos, "Block-iterative frequency-domain methods for Maxwell's equations in a plane wave basis," Opt. Express 8, 173-190 (2001).

22. J.-H. Klein-Wiele and P. Simon, "Fabrication of periodic nanostructures by phase-controlled multiple-beam interference," Appl. Phys. Lett. 83, 4707-4709 (2003). 